\title{
"I Did Not Want to Give Birth to a Child Who has HIV": Experiences Using PrEP During Pregnancy Among HIV-Uninfected Kenyan Women in HIV-Serodiscordant Couples
}

\author{
Jillian Pintye, MPH, PhD, * $†$ Kristin M. Beima-Sofie, PhD, * Grace Kimemia, BA, $\neq$ Kenneth Ngure, PhD, $\S$ \\ Susan Brown Trinidad, MA,\| Renee A. Heffron, MPH, PhD, * Jared M. Baeten, MD, PhD, * $\$ \#$ \\ Josephine Odoyo, MPH, ** Nelly Mugo, MD, ** Elizabeth A. Bukusi, MD, PhD, ${ }^{* *}$ \\ Maureen C. Kelley, PhD, $\uparrow$ t and Grace C. John-Stewart, MD, PhD*
}

Objectives: The perceptions, motivations, and beliefs of HIVuninfected women about pre-exposure prophylaxis (PrEP) use during pregnancy can influence its uptake and adherence. This study elicited the views of HIV-uninfected women with personal experience taking PrEP during pregnancy.

Received for publication April 6, 2017; accepted July 24, 2017.

From the Departments of *Global Health; and †Nursing, University of Washington, Seattle, WA; †Partners in Health Research and Development, Thika, Kenya; §Jomo Kenyatta University of Agriculture and Technology, Nairobi, Kenya; Departments of $\|$ Bioethics and Humanities; $₫$ Epidemiology; and \#Medicine, University of Washington, Seattle, WA; **Kenya Medical Research Institute, Nairobi, Kenya; and $\dagger$ † Nuffield Department of Population Health, The Ethox Centre, University of Oxford, Oxford, United Kingdom.

This study was funded through the National Institutes of Health Grant (T32AI07140 to J.P., K24 HD054314 to G.C.J.-S.) and received assistance from the University of Washington Center for AIDS Research (P30 AI27757) and the University of Washington's Global Center for Integrated Health of Women Adolescents and Children (Global WACh). The Partners Demonstration Project was funded by the US National Institutes of Mental Health (R01 MH095507), the Bill and Melinda Gates Foundation Grants (OPP47674, OPP1056051), and the US Agency for International Development (contract AID-OAA-A-12-00023). Global health bioethics research at The Ethox Centre, University of Oxford, is supported by a Wellcome Trust Strategic Award (096527).

The authors have no conflicts of interest to disclose.

J.P. and K.M.B.-S. wrote the manuscript. G.C.J.-S. and J.M.B. were the principal investigators of this study and parent study, respectively, and oversaw manuscript preparation. G.C.J.-S. and M.C.K. conceived of and designed the substudy. K.M.B.-S., M.C.K., and S.B.T. designed the interview guides. G.K., K.M.B.-S., K.N., and S.B.T. conducted interviews. G.K., J.P., K.M.B.-S., K.N., and S.B.T. analyzed the data. All authors reviewed and provided comments on the results and final manuscript.

Supplemental digital content is available for this article. Direct URL citations appear in the printed text and are provided in the HTML and PDF versions of this article on the journal's Web site (www.jaids.com).

Correspondence to: Jillian Pintye, RN, MPH, PhD, Department of Global Health, University of Washington, 325 Ninth Avenue, Box 359909, Seattle, WA 98104 (e-mail: jpintye@uw.edu).

Copyright $(2017$ The Author(s). Published by Wolters Kluwer Health, Inc. This is an open-access article distributed under the terms of the Creative Commons Attribution-Non Commercial License 4.0 (CCBY-NC), where it is permissible to download, share, remix, transform, and buildup the work provided it is properly cited. The work cannot be used commercially without permission from the journal.
Design: Qualitative interviews were conducted with HIVuninfected women who had personal experience taking PrEP while pregnant.

Methods: Semistructured interviews were conducted with 21 HIVuninfected Kenyan women in HIV-serodiscordant couples enrolled in an open-label PrEP demonstration project who became pregnant while using PrEP and continued PrEP through their pregnancy. Interviews were audio-recorded and transcribed into English. A qualitative descriptive analysis was performed, using a constant comparison approach to identify key themes related to PrEP use in pregnancy.

Results: Desire to remain HIV uninfected and have an HIV-free infant were strong motivators influencing continued use of PrEP during pregnancy. Supporting HIV-infected partners and childbearing within an HIV-serodiscordant relationship were also motivators. Women had challenges distinguishing normal pregnancy symptoms from PrEP side effects and were concerned that observed side effects could be signs of danger for the infant related to PrEP exposure. Health care providers were important conduits of knowledge about PrEP, and continuity of PrEP providers throughout pregnancy facilitated adherence.

Conclusions: HIV-uninfected women in HIV-serodiscordant couples were motivated to use PrEP during pregnancy to remain HIV uninfected and to have an HIV-free child but had concerns about side effects. Health care providers will be important for PrEP messaging and adherence support in this unique population.

Key Words: obstetrics, gynecology, PrEP, women, Africa, qualitative data, prevention of mother-to-child transmission, vertical transmission, prevention of sexual transmission

(J Acquir Immune Defic Syndr 2017;76:259-265)

\section{INTRODUCTION}

Women in high HIV prevalence regions of sub-Saharan Africa have substantial risk of acquiring HIV during and soon after pregnancy. ${ }^{1-3}$ Pregnant women who become acutely 
infected with HIV are estimated to account for $26 \%$ of all mother-to-child HIV transmissions (MTCT) in high HIV prevalence settings. ${ }^{4,5}$ Pre-exposure prophylaxis (PrEP) decreases HIV incidence in adherent women. ${ }^{6-10}$ Both the United States Centers for Disease Control and Prevention and the World Health Organization recommend PrEP for individuals at substantial HIV risk (HIV incidence $>3 \%$ ), which includes pregnant and breastfeeding women in high-burden settings. ${ }^{11,12}$ Several individual studies ${ }^{13-18}$ and one systematic review ${ }^{19}$ concluded that there is no safety-related rationale for prohibiting PrEP during pregnancy and breastfeeding. Clinical guidelines from the Centers for Disease Control and Prevention suggest continuing PrEP for women in HIV-serodiscordant partnerships who become pregnant or in those who do not know the HIV status of their male partner. ${ }^{12}$ Programmatic delivery of PrEP for pregnant women is currently being considered in high-prevalence regions. ${ }^{20}$

PrEP programmatic implementation involves awareness regarding PrEP, HIV testing, PrEP initiation, retention, and adherence, and routine monitoring. Opportunities exist to leverage existing maternal child health $(\mathrm{MCH})$ systems for PrEP delivery. Public-sector MCH clinic infrastructure in many countries with high HIV burden serves most women who become pregnant and includes frequent follow-up postpartum. Women are routinely offered HIV testing in antenatal clinics, which can identify women who would benefit from PrEP. MCH facilities are also equipped for administration of antiretrovirals, giving staff experience counseling about potential side effects and adherence to antiretrovirals. $^{21}$ The combination of substantial HIV incidence during pregnancy, increased MTCT associated with acute maternal HIV, and preexisting widespread HIV programs within $\mathrm{MCH}$ systems makes this an attractive venue for PrEP implementation. ${ }^{22}$

Before broad programmatic delivery of PrEP to pregnant women, it is important to understand motivations and beliefs for using PrEP during pregnancy to address concerns unique to this population. The personal experiences of women with direct exposure to PrEP during pregnancy offer valuable insights for informing development of effective PrEP messaging strategies and programs. To date, there have been no evaluations regarding PrEP use during pregnancy from the perspective of women who took PrEP while pregnant. We explored experiences of using PrEP during pregnancy among HIV-uninfected Kenyan women in HIVserodiscordant couples who became pregnant while using PrEP and continued PrEP use throughout their pregnancy.

\section{METHODS}

\section{Study Design and Population}

From October 2015 to March 2016, we conducted individual interviews with HIV-uninfected women in heterosexual HIV-serodiscordant couples participating in the Partners Demonstration Project at the Thika and Kisumu, Kenya sites. Thika is a peri-urban site $45 \mathrm{~km}$ north of Nairobi, Kenya where Kikuyu culture is prominent, and Kisumu is an urban site that borders Lake Victoria where Luo culture is prominent. The Partners Demonstration Project is a recently completed open-label implementation project evaluating integrated delivery of PrEP and antiretroviral therapy (ART) for HIV prevention among 1013 high-risk HIV-serodiscordant couples at 4 sites in Kenya and Uganda. ${ }^{23,24}$ Recruitment and procedures of the parent study have been previously described. ${ }^{24}$ Briefly, PrEP was recommended for HIVuninfected partners until HIV-infected partners initiated and sustained ART use for at least 6 months. All participants were members of a mutually disclosed HIV-serodiscordant couple, 18 years or older, and not using PrEP or ART at enrollment. For individuals who were willing to initiate PrEP and medically eligible, PrEP was provided for the duration of the parent study at no cost. Pregnancy testing was conducted when clinically indicated, and HIV-uninfected pregnant women were counseled about the risks and benefits of PrEP use during pregnancy and made a choice about its continuation or discontinuation. Women who continued PrEP attended monthly clinic visits through the duration of their pregnancy and discontinued PrEP after delivery.

\section{Recruitment}

All Kenyan HIV-uninfected women enrolled in the Partners Demonstration Project who became pregnant while using PrEP and were offered the opportunity to continue PrEP through their pregnancy were purposively recruited for the qualitative substudy. Overall, 30 women in the Partners Demonstration Project were elected to use PrEP during pregnancy across all sites. Of those, 21 women were from study sites in Kenya and were invited to participate in interviews; the other 9 women were from Ugandan sites and were not included in this qualitative study because of funding and logistical constraints. Women were recruited by phone or upon arrival for routine study visits by study recruitment staff members. All participants were HIV uninfected and had delivered at the time of enrollment into the qualitative study.

The parent study and this qualitative substudy received approval from the Kenya Medical Research Institute and the University of Washington ethics review boards. All participants provided written informed consent.

\section{Data Collection}

Semistructured interview guides containing open-ended questions were developed collaboratively between study team members (K.M.B.-S., S.B.T., M.C.K., K.N., G.K.) based on literature reviews and experiences in HIV prevention research. Interviewers piloted guides with Kenyan investigators and staff to ensure cultural appropriateness and clarity of questions. Guides were not piloted with women who took PrEP during pregnancy to ensure that all potential women participants were included in the interviews. However, guides were revised slightly after the first 2 interviews with women to help improve question clarity. Final guides were translated into Kiswahili and DhoLuo. Interview guides beliefs and experiences related to the main topic areas of: (1) research participation, (2) medication use and decision-making during pregnancy and breastfeeding, (3) HIV risk and prevention, (4) pregnancy 
decision-making, and (5) PrEP use during pregnancy. Detailed information on the specific questions asked and prespecified probes can be found in the interview guide (Supplemental Digital Content, http://links.lww.com/QAI/B68).

Four female and 1 male Kenyan social scientists with experience conducting in-depth interviews were recruited as interviewers. The interviewers were not involved in providing clinical or counseling services for any of the participants as part of the parent study (the Partners Demonstration Project). Before conducting interviews, all interviewers were trained on the science behind PrEP, the goal and design of the parent study, and the objectives of the substudy. Interviewers were instructed to remain fully neutral throughout the interviews. Interviewers informed interview participants that no information from the interviews would be shared with staff from the parent study or the clinic and their participation would not affect their clinical care.

Each woman participated in one individual interview. Interviews were conducted in Kiswahili, DhoLuo, or English based on interviewee preference and audio-recorded. Interviewers probed participants with prespecified and responsedriven probes to expand on their experiences to provide the richest data possible. Interviews lasted an average of $36 \mathrm{mi}-$ nutes and were conducted in a quiet, confidential area of the clinics where participants received PrEP services with only the participant and interviewer present. Interviewers took detailed notes during each interview and wrote memos after the interview. Interviews were transcribed by the interviewers continuously throughout the data collection process and were translated to English when necessary.

\section{Data Analysis}

We performed a descriptive analysis using a modified version of the constant comparison method ${ }^{25}$ to produce a description of key concepts and themes arising within and between the individual primary categories represented in the interview guides. An initial codebook was developed both deductively from the interview guide and inductively from the transcripts (by K.M.B.-S., S.B.T., K.N., G.K., and J.P.). The codebook was iteratively refined through preliminary coding applications and group discussions. Transcripts were imported into ATLAS.ti v.7 (Scientific Software Development GmbH, Berlin, Germany) for data management and analysis. All transcripts were coded independently by one member of the study team (G.K., J.P., K.M.B.-S.) using the final version of the codebook and their applied codes were reviewed by another member (G.K., J.P., K.M.B.-S.). Disagreements in code application were resolved through discussion until consensus was reached. After all data were coded, investigators used an iterative process of reading transcripts, comparing and contrasting coding, and identifying convergent and divergent themes within and between transcripts.

\section{RESULTS}

Twenty-one HIV-uninfected women who became pregnant while using PrEP participated in the study (Table 1). Almost all women were legally married (86\%) and the mean age was 27 (range, 20-36) years; 29\% were having their first
TABLE 1. Demographic Characteristics of Participants at Enrollment Into the Parent Study

\begin{tabular}{lc}
\hline & N (\%) or Mean (Range), $\mathbf{n}=\mathbf{2 1}$ \\
\hline Age, yr & $27(20-36)$ \\
Currently married & $18(86)$ \\
No. living children & $1(0-6)$ \\
Completed education, yr & $9(2-16)$ \\
Electricity in the home & $16(76)$ \\
Running water in the home & $2(10)$ \\
No. people in household & $3(2-6)$ \\
No. rooms in house & $2(1-7)$ \\
\hline
\end{tabular}

pregnancy. Three major themes emerged from the interviews related to PrEP use during pregnancy: (1) motivation for PrEP initiation and use during pregnancy, (2) the role of medication side effects and safety concerns on PrEP use, and (3) adherence challenges and successes.

PrEP was a way to maintain HIV-serodiscordant partnerships and support HIV-uninfected male partners. Women described initiating PrEP, when faced with HIV serodiscordance, as a way of ensuring the stability of their relationship and affirming their love and support for the HIV-infected partner. Having PrEP as an option gave the HIV-infected partner time to accept his HIV status and initiate ART while providing protection from transmission for the woman, allowing her to feel secure in the decision to stay in the relationship. Willingness to initiate PrEP was viewed as a way to demonstrate encouragement for HIV-infected partners to begin ART.

"The most important thing that made me to join
[the Partners Demonstration Project] was
because this guy tested positive and I didn't want
to leave him in a state where he could lose his
life. He would have decided not to use [ART]
because he was a very difficult person ... he
would have just continued drinking [alcohol] the
way he was drinking ... When I decided [to start
PrEP] he saw there is someone who cares. He
said, "Let me just join too' [by taking ART]" (21-
year-old woman)

Women also felt that continuing PrEP during pregnancy supported their HIV-infected male partners in ART adherence in the longer term. This took different forms, from creating a feeling of being in this together to more tangible support that included taking medications at the same time.

"I was taking [PrEP] to motivate my husband to take ART. We set our medication time to be the same so we take medicine together. I would take PrEP and he also takes ART and he would see that we are taking the drugs together." (24-year-old woman)

\section{PrEP Was a Way to Fulfill Pregnancy Desires While Keeping Women and Infants HIV-Free}

Women reported that initiating PrEP was the impetus for fulfilling pregnancy desires within HIV-serodiscordant 
partnerships without fear or worry of HIV transmission. Before learning about PrEP, women relied on condoms for HIV prevention and believed they would be unable to have children with their HIV-infected male partners, which was viewed as a threat to their relationship viability.

Initiating PrEP for HIV-uninfected partners and ART for HIV-infected partners before pregnancy was seen by women as a collaborative process because both partners worked together to ensure protection against HIV for the infant. Women described attempting pregnancy only postPrEP and ART initiation because they felt adequately protected from HIV transmission at that time.

"We thought that once I get on the Truvada that [becoming pregnant] should be our first objective because we were seeing that was the only way we were going to sustain the marriage, because you know men, they always want kids. So I decided ... we decided actually, the two of us ... that we take the Truvada and once we are at good [protection] levels, we could have our baby." (22-year-old woman)

Once pregnant, the primary concern for most participants shifted from personal prevention to preventing HIV for their unborn child. Most women expressed that the desire for their infants to remain HIV-free was a stronger motivation to continue PrEP during pregnancy than preventing HIV for themselves. Even when condoms were regularly used for HIV prevention, PrEP was seen as an important back-up strategy to ensure infants would be born HIV uninfected.

"I did not want to give birth to a child who has $H I V . .$. When you are pregnant and in your own house with your husband, you must make love. And even though we used condoms, sometimes they just don't put it on properly and at times, it just gets out during sexual intercourse. That was also another reason that motivated me to continue using Truvada [during pregnancy], that in case of anything, Truvada was going to help me during the pregnancy." (26-year-old woman)

The experience of taking PrEP during pregnancy and remaining HIV uninfected instilled a strong belief that PrEP was effective in preventing HIV. If given the opportunity, women in this study would use PrEP again during pregnancy.

"I have used [PrEP] and I haven't seroconverted as they maybe thought someone [in an HIV-serodiscordant couple] could ... I would use it again and again because I think it is effective..." (22-year-old woman)

\section{Women had Concerns Over PrEP Side Effects and Safety}

Few women reported any experience of side effects related to PrEP use, and almost all accounts of side effects were limited to early stages of PrEP initiation. Women expressed that the lack of adverse side effects during pregnancy affirmed their belief that PrEP was safe for their unborn infant and that PrEP was helping them.

"I have not experienced any side effect so I cannot speak about its [PrEP's] disadvantages. I can only talk about the benefits because I have used it and know how good it is. I have only experienced the beauty of it." (27-yearold woman)

Dizziness, nausea, vomiting, headaches, and feeling tired were common among women who reported experiencing any side effects while using PrEP during pregnancy. Some women recognized the similarity between pregnancy symptoms and side effects of PrEP and believed one may exaggerate the other. This was seen as a potential barrier to continuing PrEP through pregnancy.
"Now if I am pregnant and I am taking [PrEP], it could exaggerate my pregnancy symptoms. If it worsens the symptoms [of pregnancy] like nausea and the drug also has nausea as a side effect, it's a challenge. It [could] make someone to stop the dose." (22-year-old woman)

Discerning pregnancy symptoms from PrEP side effects caused confusion and distress in some women as they feared their symptoms could be signs of potential danger to their infant from PrEP use. Unilaterally, women respected health care providers as knowledgeable and trustworthy conduits of information about PrEP and its side effects. Women found that discussing PrEP use with providers helped them to feel safe and confident with their choice to continue PrEP during pregnancy.

"The most important thing was their [health
providers'] thoughts. When I got pregnant, I
had a sign and I was actually shocked. I called
the clinic. My legs were swelling and itchy. I
called immediately to inform them and inquire
whether it was an undesirable side effect [of
PrEP]. They asked me to come [to the clinic].
They told me that the most likely cause was the
pregnancy because when I started taking Truvada,
I did not experience any side effects. My legs
started swelling when I got pregnant. But it
healed on its own. So they just encouraged me
to continue using the drug and true to their word,
it did not affect me." (27-year-old woman)

Among the few women who experienced side effects from PrEP, some struggled with balancing whether the benefit of using PrEP during pregnancy was worth tolerating side effects. This concern was strengthened by the perception that using PrEP was not necessary for personal treatment and was only for prevention. However, even when symptoms were severe, most women felt the benefit of having a healthy, HIVfree infant outweighed PrEP side effects.

"These drugs [PrEP] made me sick. I kept
thinking that I have no [HIV] virus and these
drugs are making me sick. I was asking myself 
whether I was going to really be fine. I was asking myself every single day, 'Why I am on medication yet I am not sick?' I concluded that the day that I will see my child physically is the day that I will be convinced." (32-yearold woman)

Women also worried that fetal exposure to PrEP could lead to pregnancy loss or harm their newborn. Some women felt that PrEP use may be less safe during breastfeeding while the infant is growing and eating what the mother eats via breastmilk. However, most women expressed equal concern about the safety of PrEP use during pregnancy and breastfeeding.

"The pregnant woman carries a baby in her womb. What she eats is the same thing that her child will eat. Likewise with the breastfeeding mother... You have to ask yourself, maybe this baby of mine that is still in the womb can get miscarried or die [because of taking PrEP]. Also with the woman who is breastfeeding. Maybe this child she is carrying, if she eats the drug it can affect the baby, so they will have thoughts or concerns [about using PrEP]" (20-yearold woman)

In all cases, women reported that their own experience of having a healthy infant after using PrEP absolved their safety concerns.

"I didn't see any side effects on my baby and he is still ok. That is what made me to know that there is no way it [PrEP] will affect me health-wise." (21-year-old woman)

\section{Health Providers Have a Positive Influence on Adherence to PrEP}

Some women found remembering to take PrEP daily to be a challenge while others expressed that adherence was not difficult for them. Almost all women recognized that women in HIV-serodiscordant couples would be highly motivated to adhere to PrEP. However, women anticipated adherence would be a challenge if PrEP were offered to all pregnant and breastfeeding women who may not be aware of their HIV risk.

Women viewed health care providers as having an important role in facilitating adherence. Continuity between the health care providers who counseled women on PrEP before and throughout pregnancy supported continuation of PrEP adherence for some women. Health providers who valued the sensitivity of the information being discussed and maintained confidentiality helped cultivate nonjudgmental, trusting relationships with women. Positive, well-established supportive relationships with health care providers facilitated PrEP adherence throughout pregnancy and beyond.

"Initially I was coming [for clinic visits] every 3 months but after I got pregnant, [the study staff] changed it to every one month. They are the ones who were attending my pregnancy clinic visits. They would give me and all the care required when someone is pregnant, so it encouraged me to continue [PrEP]." (20-year-old woman)

\section{DISCUSSION}

This qualitative study improves understanding of motivations for PrEP use during pregnancy for women in HIVserodiscordant couples and highlights important concerns and potential barriers for effective PrEP use in this unique population. Women were initially motivated to use PrEP to maintain their HIV-serodiscordant partnership and support their HIV-infected male partners. The primary motivation to continue PrEP during pregnancy was the desire to have an infant who was HIV-free. Despite being experienced with PrEP before pregnancy, participants described uncertainties and confusion in discerning normal pregnancy symptoms from side effects of PrEP. Health care providers served as a critical support system for women while using PrEP. As programs consider wider implementation of PrEP to pregnant women at risk for HIV, there is an opportunity to use personal experiences from women who have already used PrEP during pregnancy to improve messaging for pregnant women in the future. Results from this study demonstrate the importance of developing messages that appropriately emphasize the benefits of HIV prevention for mothers and infants while simultaneously acknowledging and addressing concerns of side effects and safety.

Integration of PrEP counseling into routine maternal and child health and antenatal care settings provides an opportunity to introduce PrEP as a female-controlled HIV prevention strategy, especially to women who may be unaware of their male partner's HIV status or unable to negotiate safe sex. Our participants underscored the important role of health care providers in addressing concerns and supporting adherence. Discussion of PrEP with health care providers early and frequently in antenatal care may help address concerns about side effects and safety within a supportive patient-provider relationship. Provider-initiated PrEP counseling for pregnant women may also provide an important entry point for addressing other issues in this population, such as male involvement in antenatal care and male partner HIV testing. ${ }^{26,27}$

Some common symptoms of the first trimester of pregnancy overlap with potential side effects of PrEP initiation, including nausea, fatigue, dizziness, and gastrointestinal alterations. In our study, the experience of side effects was a barrier to PrEP continuation and adherence during pregnancy. Women in a first pregnancy have no previous experience with pregnancy symptoms, making it challenging to distinguish between PrEP and pregnancy symptoms. Women who initiate PrEP early in pregnancy may be more likely to confuse pregnancy symptoms with side effects related to PrEP. PrEP initiation before becoming pregnant, as was the case with our population, would reduce some of these concerns. Future studies among women who initiate PrEP during pregnancy should evaluate whether side effects and gestational age at PrEP initiation influences patterns of PrEP usage. 
There is some evidence that women are more motivated to address health issues during pregnancy to protect their infants ${ }^{28}$ and may find it easier to adhere to strategies, like PrEP, during this period. Several studies among HIV-infected women have found that adherence to ART for HIV treatment wanes after perceived risk of MTCT decreases postpartum. ${ }^{29-31}$ A similar waning of adherence may occur for PrEP; however, there are no data regarding this to date. In our study, women were highly motivated to continue PrEP during pregnancy to ensure their infant would be HIV-free. Adherence to PrEP may be reduced postpartum if perception of the infant's risk of HIV is also reduced. Longitudinal adherence data from women who initiate PrEP in pregnancy through postpartum could inform development of effective messaging to support PrEP adherence beyond pregnancy.

Our study has limitations. Interviews with women who used PrEP during pregnancy were conducted after delivery. Women's concerns about the safety of using PrEP during pregnancy could be influenced by positive recall bias. Future studies should evaluate safety concerns during pregnancy when the birth outcomes are unknown. All participants were in mutually disclosed HIV-serodiscordant couples and expressed strong motivation for preventing HIV. Up to $80 \%$ of pregnant African women are unaware of their partner's HIV status and therefore our data cannot be generalized to all pregnant women at risk for HIV. ${ }^{32-34}$ Our sample was limited by the number of Kenyan women who continued using PrEP through pregnancy in the Partners Demonstration Project. Future work should include larger samples of pregnant women, including those who do not know their male partner's HIV status and who may be less motivated to use PrEP or less able to navigate discreet use of PrEP.

\section{CONCLUSIONS}

PrEP holds tremendous promise as a female-controlled prevention strategy for pregnant women at high risk of HIV infection, during a time when HIV prevention has dual benefits for mothers and infants. In our study, HIVuninfected women in HIV-serodiscordant couples were motivated to use PrEP during pregnancy but had concerns unique to the period of pregnancy. Health care providers will be important for PrEP messaging and supporting women on PrEP as programmatic delivery scales up.

\section{ACKNOWLEDGMENTS}

The authors would like to thank the participants for their contributions and the Kizazi working group of the Global Center for Integrated Health of Women, Adolescents and Children (Global WACh), which provided input. The authors thank members of the larger Partners Demonstration Project team for assistance in designing and conducting this project.

\section{REFERENCES}

1. Drake AL, Wagner A, Richardson B, et al. Incident HIV during pregnancy and postpartum and risk of mother-to-child HIV transmission a systematic review and meta-analysis. PLoS Med. 2014;11:e1001608.
2. Silasi M, Cardenas I, Kwon JY, et al. Viral infections during pregnancy. Am J Reprod Immunol. 2015;73:199-213.

3. Racicot K, Kwon JY, Aldo P, et al. Understanding the complexity of the immune system during pregnancy. Am J Reprod Immunol. 2014;72:107-116.

4. Johnson LF, Stinson K, Newell ML, et al. The contribution of maternal HIV seroconversion during late pregnancy and breastfeeding to motherto-child transmission of HIV. J Acquir Immune Defic Syndr. 2012;59: 417-425.

5. Dinh TH, Delaney KP, Goga A, et al. Impact of maternal HIV seroconversion during pregnancy on early mother to child transmission of HIV (MTCT) measured at 4-8 Weeks postpartum in South Africa 20112012: a national population-based evaluation. PLoS One. 2015;10:e0125525.

6. Baeten JM, Celum C. Antiretroviral preexposure prophylaxis for HIV prevention. $N$ Engl J Med. 2013;368:83-84.

7. Thigpen MCKP, Smith DK, Segolodi TM, et al. Daily Oral Antiretroviral Use for the Prevention of HIV Infection in Heterosexually Active Young Adults in Botswana: Results From the TDF2 Study. 6th International AIDS Conference on HIV Pathogenesis, Treatment and Prevention, Rome, Italy, July 2011.

8. Grant RM, Lama JR, Anderson PL, et al. Preexposure chemoprophylaxis for HIV prevention in men who have sex with men. N Engl J Med. 2010; 363:2587-2599.

9. Choopanya K, Martin M, Suntharasamai P, et al. Antiretroviral prophylaxis for HIV infection in injecting drug users in Bangkok, Thailand (the Bangkok Tenofovir Study): a randomised, double-blind, placebocontrolled phase 3 trial. Lancet. 2013;381:2083-2090.

10. Van Damme L, Corneli A, Ahmed K, et al. Preexposure prophylaxis for HIV infection among African women. N Engl J Med. 2012;367:411-422.

11. World Health Organization. Consolidated Guidelines on the Use of Antiretroviral Drugs for Treating and Preventing HIV Infection. Geneva, Switzerland: WHO; 2016.

12. US Public Health Service. Pre-exposure Prophylaxis for the Prevention of HIV Infection in the United States-A Clinical Practice Guideline. Centers for Disease Control and Prevention. Atlanta, GA; 2014.

13. Pintye J, Langat A, Singa B, et al. Maternal tenofovir disoproxil fumarate use in pregnancy and growth outcomes among HIV-exposed uninfected infants in Kenya. Infect Dis Obstet Gynecol. 2015;2015:276851.

14. Ransom CE, Huo Y, Patel K, et al. Infant growth outcomes after maternal tenofovir disoproxil fumarate use during pregnancy. J Acquir Immune Defic Syndr. 2013;64:374-381.

15. Mugo NR, Hong T, Celum C, et al. Pregnancy incidence and outcomes among women receiving preexposure prophylaxis for HIV prevention: a randomized clinical trial. JAMA. 2014;312:362-371.

16. Siberry GK, Williams PL, Mendez H, et al. Safety of tenofovir use during pregnancy: early growth outcomes in HIV-exposed uninfected infants. AIDS. 2012;26:1151-1159.

17. Gibb DM, Kizito H, Russell EC, et al. Pregnancy and infant outcomes among HIV-infected women taking long-term ART with and without tenofovir in the DART trial. PLoS Med. 2012;9:e1001217.

18. Vigano A, Mora S, Giacomet V, et al. In utero exposure to tenofovir disoproxil fumarate does not impair growth and bone health in HIVuninfected children born to HIV-infected mothers. Antivir Ther. 2011;16: $1259-1266$

19. Mofenson LM, Baggaley RC, Mameletzis I. Tenofovir disoproxil fumarate safety for women and their infants during pregnancy and breastfeeding: systematic review. AIDS. 2017:31:213-232.

20. Ministry of Health NASCP. Guidelines on Use of Antiretroviral Drugs for Treating and Preventing HIV Infection in Kenya 2016. NASCOP; Nairobi, Kenya: 2016.

21. Gourlay A, Birdthistle I, Mburu G, et al. Barriers and facilitating factors to the uptake of antiretroviral drugs for prevention of mother-to-child transmission of HIV in sub-Saharan Africa: a systematic review. $J$ Int AIDS Soc. 2013;16:18588.

22. Price JT, Wheeler SB, Stranix-Chibanda L, et al. Cost-effectiveness of preexposure HIV prophylaxis during pregnancy and breastfeeding in subsaharan Africa. J Acquir Immune Defic Syndr. 2016;72(suppl 2):S145-S153.

23. Baeten J, Heffron R, Kidoguchi L, et al. Near Elimination of HIV Transmission in a Demonstration Project of PrEP and ART (Abstract \#24). Conference on Retroviruses and Opportunistic Infections (CROI); Seattle, WA: 2015.

24. Baeten JM, Heffron R, Kidoguchi L, et al. Integrated delivery of antiretroviral treatment and pre-exposure prophylaxis to HIV-1- 
serodiscordant couples: a prospective implementation study in Kenya and Uganda. PLoS Med. 2016;13:e1002099.

25. Glaser B, Strauss A. The Discovery of Grounded Theory, Strategies for Qualitative Research. Chicago, IL: Aldine Publishing Company; 1967.

26. Morfaw F, Mbuagbaw L, Thabane L, et al. Male involvement in prevention programs of mother to child transmission of HIV: a systematic review to identify barriers and facilitators. Syst Rev. 2013;2:5.

27. Ditekemena J, Koole O, Engmann C, et al. Determinants of male involvement in maternal and child health services in sub-Saharan Africa: a review. Reprod Health. 2012;9:32.

28. Verplanken B, Wood W. Interventions to break and create consumer habits. J Public Policy Marketing. 2006;25:90-103.

29. Fayorsey RN, Chege D, Wang C, et al. Mother infant retention for health (MIR4Health): study design, adaptations, and challenges with PMTCT implementation science research. J Acquir Immune Defic Syndr. 2016;72 (suppl 2):S137-S144.
30. Schnack A, Rempis E, Decker S, et al. Prevention of mother-to-child transmission of HIV in option B+ Era: uptake and adherence during pregnancy in Western Uganda. AIDS Patient Care STDS. 2016;30:110-118.

31. Elwell K. Facilitators and barriers to treatment adherence within PMTCT programs in Malawi. AIDS Care. 2016;28:971-975.

32. Bachanas P, Medley A, Pals S, et al. Disclosure, knowledge of partner status, and condom use among HIV-positive patients attending clinical care in Tanzania, Kenya, and Namibia. AIDS Patient Care STDS. 2013; $27: 425-435$.

33. Peltzer K, Jones D, Weiss SM, et al. Sexual risk, serostatus and intimate partner violence among couples during pregnancy in rural South Africa. AIDS Behav. 2013;17:508-516.

34. De Schacht C, Hoffman HJ, Mabunda N, et al. High rates of HIV seroconversion in pregnant women and low reported levels of HIV testing among male partners in Southern Mozambique: results from a mixed methods study. PLoS One. 2014;9:e115014. 